\title{
LIE-RINEHART ALGEBRAS, DESCENT, AND QUANTIZATION
}

\author{
JoHANNES HuEBSCHMANN \\ Université des Sciences et Technologies de Lille \\ U. F. R. de Mathématiques \\ CNRS-UMR 8524 \\ F-59 655 VILLENEUVE D'ASCQ, France \\ Johannes.Huebschmann@AGAT.UNIV-LILLE1.FR
}

February 24, 2003

\begin{abstract}
A Lie-Rinehart algebra $(A, L)$ consists of a commutative algebra $A$ and a Lie algebra $L$ with additional structure which generalizes the mutual structure of interaction between the algebra of smooth functions and the Lie algebra of smooth vector fields on a smooth manifold. Lie-Rinehart algebras provide the correct categorical language to solve the problem whether Kähler quantization commutes with reduction which, in turn, may be seen as a descent problem.
\end{abstract}

\section{Introduction}

The algebra of smooth functions $C^{\infty}(N)$ on a smooth manifold $N$ and its Lie algebra of smooth vector fields $\operatorname{Vect}(N)$ have an interesting structure of interaction. For reasons which will become apparent below, we will refer to a pair $(A, L)$ which consists of a commutative algebra $A$ and a Lie algebra $L$ with additional structure modeled on a pair of the kind $\left(C^{\infty}(N)\right.$, Vect $\left.(N)\right)$ as a Lie-Rinehart algebra. In this article we will show that the notion of Lie-Rinehart algebra provides the correct categorical language to solve a problem which we will describe shortly. Lie-Rinehart algebras occur in other areas of mathematics as well; an overview will be given in Section 1 below.

According to a philosophy going back to DIRAC, the correspondence between a classical theory and its quantum counterpart should be based on an analogy between their mathematical structures. In one direction, this correspondence, albeit not well defined, is referred to as quantization. Given a classical system with constraints which, in turn, determine what is called the reduced system, the question arises whether quantization descends to the reduced system in such a way that, once the unconstrained system has been successfully quantized, imposing the symmetries on the quantized unconstrained system is equivalent to quantizing the reduced system.

2000 Mathematics Subject Classification. $\quad$ 14L24 14L30 17B63 17B65 17B66 17B81 32C17 32C20 32Q15 32S05 32S60 53D17 53D20 53D50 58F05 58F06 81S10 .

Key words and phrases. Poisson manifolds, Poisson algebras, Poisson cohomology, holomorphic quantization, reduction and quantization, stratified Kähler spaces, geometric quantization, normal complex analytic spaces. 
This question goes back to the early days of quantum mechanics and appears already in DIRAC's work on the electron and positron [15], [16].

In the framework of Kähler quantization, the problem may be phrased as a descent problem and, indeed, under favorable circumstances which, essentially come down to requiring that the unreduced and reduced spaces be both ordinary quantizable Kähler manifolds, the problem has been known for long to have a solution [22] which, among other things, involves a version of what is referred to as KEMPF's descent lemma [46] in geometric invariant theory; see e. g. [17] and Remark 8.6 below. In the present article we will advertise the idea that the concept of Lie-Rinehart algebra provides the appropriate categorical language to solve the problem, spelled out as a descent problem, under suitable more general circumstances so that, in a sense, reduction after quantization is then equivalent to quantization after reduction; here the term "descent" should, perhaps, not be taken in too narrow a sense.

Given a classical system, its dynamical behaviour being encapsulated in a Poisson bracket among the classical observables, according to DIRAC's idea of correspondence between the classical and quantum system, the Poisson bracket should then be the classical analogue of the quantum mechanical commutator. Thus, on the physics side, the Poisson bracket is a crucial piece of structure. Mathematically, it is a crucial piece of structure as well; in particular, when the classical phase space involves singularities, these may be understood in terms of the Poisson structure. More precisely, when the classical phase space carries a stratified symplectic structure, the Poisson structure encapsulates the mutual positions of the symplectic structures on the strata. See e. g. [31], [32], [37], [39].

Up to now, the available methods have been insufficient to attack the problem of quantization of reduced observables, once the reduced phase space is no longer a smooth manifold; we will refer to this situation as the singular case. The singular case is the rule rather than the exception. For example, simple classical mechanical systems and the solution spaces of classical field theories involve singularities; see e. g. [3] and the references there. In the presence of singularities, restricting quantization to a smooth open dense part, the "top stratum", leads to a loss of information and in fact to inconsistent results, cf. Section 4 of [41]. To overcome these difficulties on the classical level, in [39], we isolated a certain class of "Kähler spaces with singularities", which we call stratified Kähler spaces. On such a space, the complex analytic structure alone is unsatisfactory for issues related with quantization because it overlooks the requisite Poisson structures. In [41] we developed the Kähler quantization scheme over (complex analytic) stratified Kähler spaces. A suitable notion of prequantization, phrased in terms of prequantum modules introduced in [30], yields the requisite representation of the Poisson algebra; in particular, this representation satisfies the Dirac condition. A suitably defined concept of stratified Kähler polarization then takes care of the irreducibility problem, as does an ordinary polarization in the smooth case. Over a stratified space, the appropriate quantum phase space is what we call a costratified Hilbert space; this is a system of Hilbert spaces, one for each stratum, which arises from quantization on the closure of that stratum, the stratification provides linear maps between these Hilbert spaces reversing the partial ordering among the strata, and these linear maps are compatible with the quantizations. The main result obtained in [41] says that, for a positive Kähler manifold with a hamiltonian action of a compact Lie group, when suitable additional 
conditions are imposed, reduction after quantization coincides with quantization after reduction in the sense that not only the reduced and unreduced quantum phase spaces correspond but the invariant unreduced and reduced quantum observables as well. Examples abound; one such class of examples, involving holomorphic nilpotent orbits and in particular angular momentum zero spaces, has been treated in [41]. A particular case thereof will be reproduced in Section 6 below, for the sake of illustration.

A stratified polarization, see Section 5 below for details, is defined in terms of an appropriate Lie-Rinehart algebra which, for any Poisson algebra, serves as a replacement for the tangent bundle of a smooth symplectic manifold. The question whether quantization commutes with reduction includes the question whether what is behind the phrase "in terms of an appropriate Lie-Rinehart algebra" descends to the reduced level. This hints at interpreting this question as a descent problem.

To our knowledge, the idea of Lie-Rinehart algebra was first used by JACOBSON in [43] (without being explicitly identified as a structure in its own) to study certain field extensions. Thereafter this idea occurred in other areas including differential geometry and differential Galois theory. More details will be given below.

I am indebted to the organizers of the meeting for having given me the chance to illustrate an application of Lie-Rinehart algebras to a problem phrased in a language entirely different from that of Lie-Rinehart algebras. Perhaps one can build a general Galois theory including ordinary Galois theory, differential Galois theory, and ordinary principal bundles, in which Lie-Rinehart algebras appear as certain objects which capture infinitesimal symmetries.

\section{Lie-Rinehart algebras}

Let $R$ be a commutative ring with 1 taken as ground ring which, for the moment, may be arbitrary. For a commutative $R$-algebra $A$, we denote by $\operatorname{Der}(A)$ the $R$-Lie algebra of derivations of $A$, with its standard Lie algebra structure. An $(R, A)$-Lie algebra [66] is a Lie algebra $L$ over $R$ which acts on (the left of) $A$ (by derivations) and is also an $A$-module satisfying suitable compatibility conditions which generalize the usual properties of the Lie algebra of vector fields on a smooth manifold viewed as a module over its ring of functions; these conditions read

$$
\begin{aligned}
{[\alpha, a \beta] } & =\alpha(a) \beta+a[\alpha, \beta], \\
(a \alpha)(b) & =a(\alpha(b)),
\end{aligned}
$$

where $a, b \in A$ and $\alpha, \beta \in L$. When the emphasis is on the pair $(A, L)$ with the mutual structure of interaction between $A$ and $L$, we refer to the pair $(A, L)$ as a Lie-Rinehart algebra. Given an arbitrary commutative algebra $A$ over $R$, an obvious example of a Lie-Rinehart algebra is the pair $(A, \operatorname{Der}(A))$, with the obvious action of $\operatorname{Der}(A)$ on $A$ and obvious $A$-module structure on $\operatorname{Der}(A)$. There is an obvious notion of morphism of Lie-Rinehart algebras and, with this notion of morphism, Lie-Rinehart algebras constitute a category. More details may be found in RineHART [66] and in our papers [29] and [30].

We will now briefly spell out some of the salient features of Lie-Rinehart algebras. Given an $(R, A)$-Lie algebra $L$, its universal algebra $\left(U(A, L), \iota_{L}, \iota_{A}\right)$ is an $R$-algebra 
$U(A, L)$ together with a morphism $\iota_{A}: A \longrightarrow U(A, L)$ of $R$-algebras and a morphism $\iota_{L}: L \longrightarrow U(A, L)$ of Lie algebras over $R$ having the properties

$$
\iota_{A}(a) \iota_{L}(\alpha)=\iota_{L}(a \alpha), \quad \iota_{L}(\alpha) \iota_{A}(a)-\iota_{A}(a) \iota_{L}(\alpha)=\iota_{A}(\alpha(a))
$$

and $\left(U(A, L), \iota_{L}, \iota_{A}\right)$ is universal among triples $\left(B, \phi_{L}, \phi_{A}\right)$ having these properties. For example, when $A$ is the algebra of smooth functions on a smooth manifold $N$ and $L$ the Lie algebra of smooth vector fields on $N$, then $U(A, L)$ is the algebra of (globally defined) differential operators on $N$. An explicit construction for the $R$-algebra $U(A, L)$ is given in Rinehart [66]. See our paper [29] for an alternate construction which employs the MASSEY-PETERSON [59] algebra.

The universal algebra $U(A, L)$ admits an obvious filtered algebra structure $U_{-1} \subseteq$ $U_{0} \subseteq U_{1} \subseteq \ldots$, cf. Rinehart [66], where $U_{-1}(A, L)=0$ and where, for $p \geq 0, U_{p}(A, L)$ is the left $A$-submodule of $U(A, L)$ generated by products of at most $p$ elements of the image $\bar{L}$ of $L$ in $U(A, L)$, and the associated graded object $E^{0}(U(A, L))$ inherits a commutative graded $A$-algebra structure. The Poincaré-Birkhoff-Witt Theorem for $U(A, L)$ then takes the following form where $S_{A}[L]$ denotes the symmetric $A$-algebra on $L$, cf. (3.1) of RineHART [66].

Theorem 1.1 [Rinehart]. For an $(R, A)$-Lie algebra $L$ which is projective as an A-module, the canonical A-epimorphism $S_{A}[L] \longrightarrow E^{0}(U(A, L))$ is an isomorphism of A-algebras.

Consequently, for an $(R, A)$-Lie algebra $L$ which is projective as an $A$-module, the morphism $\iota_{L}: L \longrightarrow U(A, L)$ is injective.

The construction of the ordinary Koszul complex computing Lie algebra cohomology carries over as well: Let $\Lambda_{A}(s L)$ be the exterior Hopf algebra over $A$ on the suspension $s L$ of $L$, where "suspension" means that $s L$ is $L$ except that its elements are regraded up by 1. RINEHART [66] has proved that the ordinary Chevalley-Eilenberg operator induces an $U(A, L)$-linear operator $d$ on $U(A, L) \otimes_{A} \Lambda_{A}(s L)$ (this is not obvious since $L$ is not an ordinary $A$-Lie algebra unless $L$ acts trivially on $A$ ) having square zero. We will refer to

$$
K(A, L)=\left(U(A, L) \otimes_{A} \Lambda_{A}(s L), d\right)
$$

as the Rinehart complex for $(A, L)$. It is manifest that the Rinehart complex is functorial in $(A, L)$. Moreover, as a graded $A$-module, the resulting chain complex $\operatorname{Hom}_{U(A, L)}(K(A, L), A)$ underlies the $A$-algebra $\operatorname{Alt}_{A}(L, A)$ of $A$-multilinear functions on $L$ but, beware, the differential is linear only over the ground ring $R$ and turns $\operatorname{Alt}_{A}(L, A)$ into a differential graded cocommutative algebra over $R$; we will refer to this differential graded $R$-algebra as the Rinehart algebra of $(A, L)$. Rinehart also noticed that, when $L$ is projective or free as a left $A$-module, $K(A, L)$ is a projective or free resolution of $A$ in the category of left $U(A, L)$-modules according as $L$ is a projective or free left $A$-module; details may be found in [66]. In particular, the Rinehart algebra $\left(\operatorname{Alt}_{A}(L, A), d\right)$ then computes the Ext-algebra $\operatorname{Ext}_{U(A, L)}^{*}(A, A)$.

Rinehart also noticed that, when $A$ is the algebra of smooth functions on a smooth manifold $N$ and $L$ the Lie algebra of smooth vector fields on $N$, then $\left.\operatorname{Alt}_{A}(L, A), d\right)\left(=\operatorname{Hom}_{U(A, L)}(K(A, L), A)\right)$ is the ordinary de Rham complex of $N$ 
whence, as an algebra, the de Rham cohomology of $N$ amounts to the Ext-algebra $\operatorname{Ext}_{U(A, L)}^{*}(A, A)$ over the algebra $U(A, L)$ of differential operators on $N$. Likewise, for a Lie algebra $L$ over $R$ acting trivially on $R, K(R, L)$ is the ordinary Koszul complex; in particular, when $L$ is projective as an $R$-module, $K(R, L)$ is the ordinary Koszul resolution of the ground ring $R$. Thus the cohomology of Lie-Rinehart algebras comprises de Rham- as well as Lie algebra cohomology. In particular, this offers a possible explanation why Chevalley and EILENBerg [12], when they first isolated Lie algebra cohomology, derived their formulas by abstracting from the de Rham operator of a smooth manifold. Suitable graded versions of the cohomology of LieRinehart algebras comprise as well Hodge cohomology and coherent sheaf cohomology of complex manifolds [36, 38].

The classical differential geometry notions of connection, curvature, characteristic classes, etc. may be developed for arbitrary Lie-Rinehart algebras [29], [30], [34], and there are notions of duality for Lie-Rinehart algebras generalizing Poincaré duality [35]; the idea of duality has been shown in [33] to cast new light on Gerstenhaberand Batalin-Vilkovisky algebras. In a sense these homological algebra interpretations of Batalin-Vilkovisky algebras push further Rinehart's observations related with the interpretation of de Rham cohomology as certain Ext-groups. Graded versions of duality for Lie-Rinehart algebras [36], [38] may be used to study e. g. complex manifolds, CR-structures, and the mirror conjecture.

Lie-Rinehart algebras were implicitly used already by JACOBSON [43] and later by Hochschild [27]. The idea of Lie-Rinehart algebra has been introduced by a very large number of authors, most of whom independently proposed their own terminology. I am indebted to K. Mackenzie for his help with compiling the following list in chronological order: Pseudo-algèbre de Lie: Herz, 1953 [24] — actually, Herz seems to be the first to describe the structure in a form which makes its generality clear-; Lie d-ring: Palais, 1961 [62]; (R,C)-Lie algebra: Rinehart, 1963 [66]; (R,C)-éspace d'Elie Cartan régulier et sans courbure: de Barros, 1964 [14]; (R,C)-algèbre de Lie: Bkouche, 1966 [7]; Lie algebra with an associated module structure: Hermann, 1967 [23]; Lie module: Nelson, 1967 [61]; Pseudo-algèbre de Lie: Pradines, 1967 [64]; (A, C) system: Kostant and Sternberg, 1971 [52]; Sheaf of twisted Lie algebras: Kamber and Tondeur, 1971 [44]; Algèbre de Lie sur C/R: Illusie, 1972 [42]; Lie algebra extension: N. Teleman, 1972 [76]; Lie-Cartan pair: Kastler and Stora, 1985 [45]; Atiyah algebra: Beilinson and Schechtmann, 1988 [6]; Lie-Rinehart algebra: Huebschmann, 1990 [29]; Differential Lie algebra: Kosmann-Schwarzbach and Magri, 1990 [50]. Hinich and Schechtman (1993) [26] have used the term Lie algebroid for the general algebraic concept. In differential Galois theory, Lie-Rinehart algebras occur under the name "algèbre différentielle" in a paper by FAHIM [18]. Lie-Rinehart algebras occur as well in Chase [10] and StashefF [75]. We have chosen to use the terminology Lie-Rinehart algebra since, as already pointed out, Rinehart subsumed the cohomology of these objects under standard homological algebra and established a Poincaré-Birkhoff-Witt theorem for them. In differential geometry, $(R, A)$-Lie algebras arise as spaces of sections of Lie algebroids. These, in turn, were introduced in 1966 by Pradines [63] and, in that paper, Pradines raised the issue whether Lie's third theorem holds for Lie algebroids in the sense that any Lie algebroid integrates to a Lie groupoid. CRAINIC and Fernandes [13] have recently given a solution of this problem in terms of suitably defined obstructions. See MAckenzIE [56] for a complete 
account of Lie algebroids and Lie groupoids, as well as CANAS DA Silva-Weinstein [9] and MACKENZIE [57] for more recent surveys on particular aspects. The idea of Lie algebroid is lurking behind a construction in FuCHSSTEINER [19] (see Remark 2.6 below). A descent construction for Lie algebroids may be found in HigGinsMACKENZIE [25]. A general notion of morphism of Lie algebroids has been introduced by Almeida-Kumpera [2]. This notion has been used by S. Chemla [11] to study notions of duality for Lie algebroids in complex algebraic geometry generalizing Serre duality. Lie-Rinehart algebras are lurking as well behind the nowadays very active research area of $D$-modules.

REMARK (out of context). At the end of his paper [66], Rinehart introduced an operator on the Hochschild complex of a commutative algebra which, some 20 years later, was reinvented by $\mathrm{A}$. Connes in order to define cyclic cohomology.

\section{Poisson algebras}

For intelligibility, we recall briefly how for an arbitrary Poisson algebra an appropriate Lie-Rinehart algebra serves as a replacement for the tangent bundle of a smooth symplectic manifold.

Let $(A,\{\cdot, \cdot\})$ be a Poisson algebra, and let $D_{A}$ be the $A$-module of formal differentials of $A$ the elements of which we write as $d u$, for $u \in A$. For $u, v \in A$, the assignment to $(d u, d v)$ of $\pi(d u, d v)=\{u, v\}$ yields an $A$-valued $A$-bilinear skewsymmetric 2 -form $\pi=\pi_{\{\cdot, \cdot\}}$ on $D_{A}$, the Poisson 2-form for $(A,\{\cdot, \cdot\})$. Its adjoint

$$
\pi^{\sharp}: D_{A} \rightarrow \operatorname{Der}(A)=\operatorname{Hom}_{A}\left(D_{A}, A\right)
$$

is a morphism of $A$-modules, and the formula

$$
[a d u, b d v]=a\{u, b\} d v+b\{a, v\} d u+a b d\{u, v\}
$$

yields a Lie bracket $[\cdot, \cdot]$ on $D_{A}$, viewed as an $R$-module. More details may be found in [29]. For the record we recall the following, established in [29] (3.8).

Proposition 2.3. The A-module structure on $D_{A}$, the bracket $[\cdot, \cdot]$, and the morphism $\pi^{\sharp}$ of A-modules turn the pair $\left(A, D_{A}\right)$ into a Lie-Rinehart algebra in such a way that $\pi^{\sharp}$ is a morphism of Lie-Rinehart algebras.

We write $D_{\{\cdot, \cdot\}}=\left(D_{A},[\cdot, \cdot], \pi^{\sharp}\right)$. The 2-form $\pi_{\{\cdot, \cdot\}}$, which is defined for every Poisson algebra, is plainly a 2-cocycle in the Rinehart algebra $\left(\operatorname{Alt}_{A}\left(D_{\{\cdot, \cdot\}}, A\right), d\right)$. In [29], we defined the Poisson cohomology $\mathrm{H}_{\text {Poisson }}^{*}(A, A)$ of the Poisson algebra $(A,\{\cdot, \cdot\})$ to be the cohomology of this Rinehart algebra, that is,

$$
\mathrm{H}_{\text {Poisson }}^{*}(A, A)=\mathrm{H}^{*}\left(\operatorname{Alt}_{A}\left(D_{\{\cdot, \cdot\}}, A\right), d\right) \text {. }
$$

Henceforth we shall take as ground ring that of the reals $\mathbb{R}$ or that of the complex numbers $\mathbb{C}$. We shall consider spaces $N$ with an algebra of continuous $\mathbb{R}$-valued or $\mathbb{C}$-valued functions, deliberately denoted by $C^{\infty}(N, \mathbb{R})$ or $C^{\infty}(N, \mathbb{C})$ as appropriate, or by just $C^{\infty}(N)$, for example ordinary smooth manifolds and ordinary smooth functions; such an algebra $C^{\infty}(N)$ will then be referred to as a smooth structure on $N$, and $C^{\infty}(N)$ will be viewed as part of the structure of $N$. A space may support different smooth structures, though. Given a space $N$ with a smooth 
structure $C^{\infty}(N)$, we shall write $\Omega^{1}(N)$ for the space of formal differentials with those differentials divided out that are zero at each point, cf. [53]; for example, over the real line with its ordinary smooth structure, the formal differentials $d \sin x$ and $\cos x d x$ do not coincide but the formal differential $d \sin x-\cos x d x$ is zero at each point. At a point of $N$, the object $\Omega^{1}(N)$ comes down to the ordinary space of differentials for the smooth structure on $N$; see Section 1.3 of our paper [41] for details. When $N$ is an ordinary smooth manifold, $\Omega^{1}(N)$ amounts to the space of smooth sections of the cotangent bundle. For a general smooth space $N$ over the reals, when $A=C^{\infty}(N, \mathbb{R})$ is endowed with a Poisson structure, the formula (1.2) yields a Lie-bracket $[\cdot, \cdot]$ on the $A$-module $\Omega^{1}(N)$ and the 2 -form $\pi_{\{\cdot, \cdot\}}$ is still defined on $\Omega^{1}(N)$; its adjoint then yields an $A$-linear map $\pi^{\sharp}$ from $\Omega^{1}(N)$ to $\operatorname{Der}(A)$ in such a way that $\left([\cdot, \cdot], \pi^{\sharp}\right)$ is an $(\mathbb{R}, A)$-Lie algebra structure on $\Omega^{1}(N)$ and that the adjoint $\pi^{\sharp}$ is a morphism of $(\mathbb{R}, A)$-Lie algebras. Here the notation $[\cdot, \cdot], \pi^{\sharp}, \pi_{\{\cdot, \cdot\}}$ is abused somewhat. The obvious projection map from $D_{C^{\infty}(N)}$ to $\Omega^{1}(N)$ is plainly compatible with the Lie-Rinehart structures. The fact that $D_{C^{\infty}(N)}$ is "bigger" than $\Omega^{1}(N)$ in the sense that the surjection from the former to the latter has a non-trivial kernel causes no problem here since the $A$-dual of this surjection, that is, the induced map from $\operatorname{Hom}\left(\Omega^{1}(N), A\right)$ to $\operatorname{Hom}\left(D_{C^{\infty}(N)}, A\right)$, is an isomorphism. We shall write $\Omega^{1}(N)_{\{\cdot, \cdot\}}=\left(\Omega^{1}(N),[\cdot, \cdot], \pi^{\sharp}\right)$. When $N$ is a smooth manifold in the usual sense, the range $\operatorname{Der}(A)$ of the adjoint map $\pi^{\sharp}$ from $\Omega^{1}(N)$ to $\operatorname{Der}(A)$ boils down to the space $\operatorname{Vect}(N)$ of smooth vector fields on $N$. In this case, the Poisson structure on $N$ is symplectic, that is, arises from a (uniquely determined) symplectic structure on $N$, if and only if $\pi^{\sharp}$, which may now be written as a morphism of smooth vector bundles from $\mathrm{T}^{*} N$ to $\mathrm{T} N$, is an isomorphism.

The 2-form $\pi_{\{\cdot, \cdot\}}$ is a 2-cocycle in the Rinehart algebra $\left(\operatorname{Alt}_{A}\left(\Omega^{1}(N)_{\{\cdot, \cdot\}}, A\right), d\right)$ and the canonical map of differential graded algebras from $\left(\operatorname{Alt}_{A}\left(\Omega^{1}(N)_{\{\cdot, \cdot\}}, A\right), d\right)$ to $\left.\operatorname{Alt}_{A}\left(D_{\{\cdot, \cdot\}}, A\right), d\right)$ is an isomorphism. In particular, we may take the cohomology of the Rinehart algebra $\left(\operatorname{Alt}_{A}\left(\Omega^{1}(N)_{\{\cdot, \cdot\}}, A\right), d\right)$ as the definition of the Poisson cohomology $\mathrm{H}_{\text {Poisson }}^{*}(A, A)$ of the Poisson algebra $(A,\{\cdot, \cdot\})$ as well. When $N$ is an ordinary smooth manifold, its algebra of ordinary smooth functions being endowed with a Poisson structure, this notion of Poisson cohomology comes down to that introduced by Lichnerowicz [55]. For a general Poisson algebra $A$, the 2-form $\pi_{\{\cdot, \cdot\}}$, be it defined on $\Omega^{1}(N)_{\{\cdot, \cdot\}}$ for the case where $A$ is the structure algebra $C^{\infty}(N)$ of a space $N$ or on $D_{\{\cdot, \cdot\}}$ for an arbitrary Poisson algebra, generalizes the symplectic form of a symplectic manifold; see Section 3 of [29] for details. Suffice it to make the following observation, relevant for quantization: Consider a space $N$ with a smooth structure $C^{\infty}(N)$ which, in turn, is endowed with a Poisson bracket $\{\cdot, \cdot\}$. The Poisson 2-form $\pi_{\{\cdot, \cdot\}}$ determines an extension of Lie-Rinehart algebras which is central as a Lie algebra extension. For technical reasons it is more convenient to take here the extension

$$
0 \rightarrow A \rightarrow \bar{L}_{\{\cdot, \cdot\}} \rightarrow \Omega^{1}(N)_{\{\cdot, \cdot\}} \rightarrow 0
$$

which corresponds to the negative of the Poisson 2-form. Here, as $A$-modules, $\bar{L}_{\{\cdot, \cdot\}}=A \oplus \Omega^{1}(N)_{\{\cdot, \cdot\}}$, and the Lie bracket on $\bar{L}_{\{\cdot, \cdot\}}$ is given by

$$
[(a, d u),(b, d v)]=(\{u, b\}+\{a, v\}-\{u, v\}, d\{u, v\}), \quad a, b, u, v \in A
$$


Here we have written " $\bar{L}$ " rather than simply $L$ to indicate that the extension (2.4) represents the negative of the class of $\pi_{\{\cdot, \cdot\}}$ in the second cohomology group $\mathrm{H}^{2}\left(\operatorname{Alt}_{A}\left(D_{\{\cdot, \cdot\}}, A\right), d\right)$ of the corresponding Rinehart algebra, cf. [29], and the notation $d u, d v$ etc. is abused somewhat. Now, any principal circle bundle admits as its infinitesimal object an Atiyah-sequence [5] whose spaces of sections constitute a central extension of Lie-Rinehart algebras; see [56] for a complete account of Atiyahsequences and [34] for a theory of characteristic classes for extensions of general Lie-Rinehart algebras. When the Poisson structure is an ordinary smooth symplectic Poisson structure whose symplectic form represents an integral cohomology class, the Lie-Rinehart algebra extension (2.4) comes down to the space of sections of the Atiyah-sequence of the principal circle bundle classified by that cohomology class.

REMARK 2.6. For the special case where $N$ is an ordinary smooth manifold, $C^{\infty}(N)$ its algebra of ordinary smooth functions, and where $\{\cdot, \cdot\}$ is a Poisson structure on $C^{\infty}(N)$, the Lie-Rinehart structure on the pair $\left(C^{\infty}(N), \Omega^{1}(N)\right)$ (where $\Omega^{1}(N)$ amounts to the space of ordinary smooth 1 -forms on $N$ ) was discovered by a number of authors during the 80's most of whom phrased the structure in terms of the corresponding Lie algebroid structure on the cotangent bundle of $N$; some historical comments may be found in [29]. The first reference I am aware of where versions of the Lie algebroid bracket and of the anchor map may be found is [19]; in that paper, the notion of "implectic operator" is introduced-this is the operator nowadays referred to as Poisson tensor - and the Lie bracket and anchor map are the formula (2) and morphism written as $\Omega_{\phi}$, respectively, in that paper. The construction in terms of formal differentials carried out in [29] (and reproduced above) - as opposed to the Lie algebroid construction - is more general, though, since it refers to an arbitrary Poisson structure, not necessarily one which is defined on an algebra of smooth functions on an ordinary smooth manifold. In fact, the aim of the present article is to demonstrate the significance of this more general construction which works as well for Poisson algebras of continuous functions defined on spaces with singularities where among other things it yields a tool to relate the Poisson structures on the strata of a stratified symplectic space; suitably translated into the language of sheaves, it also works over not necessarily non-singular varieties.

\section{Quantization}

According to DIRAC [15], [16], a quantization of a classical system described by a real Poisson algebra $(A,\{\cdot, \cdot\})$ is a representation $a \mapsto \hat{a}$ of a certain Lie subalgebra $B$ of $A, A$ and $B$ being viewed merely as Lie algebras, by symmetric or, whenever possible, self-adjoint, operators $\hat{a}$ on a Hilbert space $\mathcal{H}$ such that (i) the Dirac condition

$$
i[\hat{a}, \hat{b}]=\widehat{\{a, b\}}
$$

holds; that (ii) for a constant $c$, the operator $\hat{c}$ is given by $\hat{c}=c \mathrm{Id}$; and that (iii) the representation is irreducible. Here the factor $i$ in the Dirac condition is forced by the interpretation of quantum mechanics: Observables are to be represented by symmetric (or self-adjoint) operators but the ordinary commutator of two symmetric operators is not symmetric. The second requirement rules out the adjoint representation, and the irreducibility condition is forced by the requiremend that phase transitions be possible between two different states. See e. g. Sniatycki [71] or Woodhouse [79]. Also it is known that for $B=A$ the problem has no solution whence the requirement 
that only a sub Lie algebra of $A$ be represented. The physical constant $\hbar$ is here absorbed in the Poisson structure. It has become common to refer to a procedure furnishing a representation that satisfies only (i) and (ii) above as prequantization.

Under suitable circumstances, over a smooth symplectic manifold, the geometric quantization scheme developed by Kirilliov [48], Kostant [51], Souriau [74], and I. SEGAL [67], furnishes a quantization; see [71] or [79] for complete accounts. We confine ourselves with the remark that geometric quantization proceeds in two steps. The first step, prequantization, yields a representation of the Lie algebra underlying the whole Poisson algebra which satisfies (i) and (ii) but such a representation is not irreducible; the second step involves a choice of polarization to force the irreducibility condition. In particular, a Kähler polarization leads to what is called Kähler quantization. The existence of a Kähler polarization entails that the underlying manifold carries an ordinary Kähler structure. In the singular case, the ordinary geometric quantization scheme is no longer available, though. In the rest of the paper we shall describe how, under certain favorable circumstances, the difficulties in the singular case can be overcome in the framework of Kähler quantization. An observation crucial in the singular case is that the notion of polarization can be given a meaning by means of appropriately defined Lie-Rinehart algebras. Before going into details, we will briefly explain one of the origins of singularities.

\section{Symmetries}

Recall that a symplectic manifold is a smooth manifold $N$ together with a closed nondegenerate 2-form $\sigma$. Given a function $f$, the identity $\sigma\left(X_{f}, \cdot\right)=d f$ then associates a uniquely determined vector field $X_{f}$ to $f$, the Hamiltonian vector field of $f$ and, given two functions $f$ and $h$, their Poisson bracket $\{f, h\}$ is defined by $\{f, h\}=X_{f} h$. This yields a Poisson bracket $\{\cdot, \cdot\}$ on the algebra $C^{\infty}(N)$ of ordinary smooth functions on $N$, referred to as a symplectic Poisson bracket.

Given a Lie group $G$, a hamiltonian $G$-space is a smooth symplectic $G$-manifold $(N, \sigma)$ together with a smooth $G$-equivariant map $\mu$ from $N$ to the dual $\mathfrak{g}^{*}$ of the Lie algebra $\mathfrak{g}$ of $G$ satisfying the formula

$$
\sigma\left(X_{N}, \cdot\right)=X \circ d \mu
$$

for every $X \in \mathfrak{g} ;$ here $X_{N}$ denotes the vector field on $N$ induced by $X \in \mathfrak{g}$ via the $G$-action, and $X$ is viewed as a linear form on $\mathfrak{g}^{*}$. The map $\mu$ is called a momentum mapping (or moment map). We recall that (4.1) says that, given $X \in \mathfrak{g}$, the vector field $X_{N}$ is the hamiltonian vector field for the smooth function $X \circ \mu$ on $N$. See e. g. [1] for details. Given a hamiltonian $G$-space $(N, \sigma, \mu)$, the space $N_{\text {red }}=\mu^{-1}(0) / G$ is called the reduced space. When $G$ is not compact, this space may have bad properties; for example, it is not even a Hausdorff space when there are non-closed $G$-orbits in $N$.

Let $\left.C^{\infty}\left(N_{\text {red }}\right)=\left(C^{\infty}(N)\right)^{G}\right) / I^{G}$, where $I^{G}$ refers to the ideal (in the algebra $\left.\left(C^{\infty}(N)\right)^{G}\right)$ of smooth $G$-invariant functions on $N$ which vanish on the zero locus $\mu^{-1}(0)$; this is a smooth structure on the reduced space $N_{\text {red }}$. As observed by ARMSCushman-Gotay [3], the Noether Theorem implies that the symplectic Poisson structure on $C^{\infty}(N)$ descends to a Poisson structure $\{,\}_{\text {red }}$ on $C^{\infty}\left(N_{\text {red }}\right)$, and SJAMAAR-LERMAN [70] have shown that, when $G$ is compact and when the momentum 
mapping is proper, the orbit type decomposition of $N_{\text {red }}$ is a stratification in the sense of GORESKY-MACPherson [20]. The idea that the orbit type decomposition is a stratification (in a somewhat weaker sense) may be found already in [4]. For intelligibility, we recall some of the requisite technical details.

A decomposition of a space $Y$ into pieces which are smooth manifolds such that these pieces fit together in a certain precise way is called a stratification [20]. More precisely: Let $Y$ be a Hausdorff paracompact topological space and let $\mathcal{I}$ be a partially ordered set with order relation denoted by $\leq$. An $\mathcal{I}$-decomposition of $Y$ is a locally finite collection of disjoint locally closed manifolds $S_{i} \subseteq Y$ called pieces (recall that a collection $\mathcal{A}$ of subsets of $Y$ is said to be locally finite provided every $x \in Y$ has a neighborhood $U_{x}$ in $Y$ such that $U_{x} \cap A \neq \emptyset$ for at most finitely many $A$ in $\mathcal{A}$ ) such that the following hold:

$$
\begin{aligned}
& Y=\cup S_{i}(i \in \mathcal{I}), \\
& S_{i} \cap \bar{S}_{j} \neq \emptyset \Longleftrightarrow S_{i} \subseteq \bar{S}_{j} \Longleftrightarrow i \leq j \quad(i, j \in \mathcal{I}) .
\end{aligned}
$$

The space $Y$ is then called a decomposed space. A decomposed space $Y$ is said to be a stratified space if the pieces of $Y$, called strata, satisfy the following condition: Given a point $x$ in a piece $S$ there is an open neighborhood $U$ of $x$ in $Y$, an open ball $B$ around $x$ in $S$, a stratified space $\Lambda$, called the link of $x$, and a decomposition preserving homeomorphism from $B \times C^{\circ}(\Lambda)$ onto $U$. Here $C^{\circ}(\Lambda)$ refers to the open cone on $\Lambda$ and, as a stratified space, $\Lambda$ is less complicated than $C^{\circ}(\Lambda)$ whence the definition is not circular; the idea of complication is here made precise by means of the notion of depth.

A stratified symplectic space [70] is a stratified space $Y$ together with a Poisson algebra $\left(C^{\infty}(Y),\{\},\right)$ of continuous functions on $Y$ which, on each piece of the decomposition, restricts to an ordinary smooth symplectic Poisson structure; in particular, $C^{\infty}(Y)$ is a smooth structure on $Y$.

EXAMPLE 4.2. On the ordinary plane, with coordinates $x_{1}, x_{2}$, consider the algebra $A$ of smooth functions in the coordinate functions $x_{1}, x_{2}$ together with, which is crucial here, an additional function $r$ which is the radius function, subject to the relation $x_{1}^{2}+x_{2}^{2}=r^{2}$. Notice that $r$ is not a smooth function in the usual sense whence the algebra $A$ is strictly larger than that of ordinary smooth functions on the plane. The Poisson structure $\{\cdot, \cdot\}$ on $A$ given by the formulas

$$
\left\{x_{1}, x_{2}\right\}=2 r, \quad\left\{x_{1}, r\right\}=2 x_{2}, \quad\left\{x_{2}, r\right\}=-2 x_{1}
$$

turns the plane into a stratified symplectic space. Geometrically, the plane is taken here as a half cone, the algebra $A$ being that of Whitney-smooth functions on the half cone, with reference to the embedding into 3 -space; there are two strata, the vertex of the half cone and the complement thereof. On the complement of the vertex, which is a punctured plane, the Poisson structure is symplectic. In physics, the Poisson algebra $(A,\{\cdot, \cdot\})$ arises, for $n \geq 2$, as the reduced Poisson algebra of a single particle in $\mathbb{R}^{n}$ with $\mathrm{O}(n, \mathbb{R})$-symmetry and angular momentum zero. For $n=1$, the example still makes sense: the symmetry group is then just a copy of $\mathbb{Z} / 2$, and the angular momentum is zero.

Given a hamiltonian $G$-space $(N, \sigma, \mu)$ with $G$ compact, in view of an observation in [70], the Arms-Cushman-Gotay construction turns $\left(N_{\text {red }}, C^{\infty}\left(N_{\text {red }}\right),\{,\}_{\text {red }}\right)$ (more 
precisely: each connected component of $N_{\text {red }}$ in case the momentum mapping is not proper) into a stratified symplectic space. When $N_{\text {red }}$ is smooth, i. e. has a single stratum, this space is just a smooth symplectic manifold, the ordinary MARSDEn-Weinstein reduced space [58].

\section{Stratified complex polarizations}

Within the ordinary geometric quantization scheme, the irreducibility requirement is taken care of by means of a polarization. In particular, a complex polarization for an ordinary symplectic manifold $N$ is an integrable Lagrangian distribution $F \subseteq \mathrm{T}^{\mathbb{C}} N$ of the complexified tangent bundle $\mathrm{T}^{\mathbb{C}} N[79]$; under the identification of $\mathrm{T}^{\mathbb{C}} N$ with its (complex) dual coming from the symplectic structure, a complex polarization $F$ then corresponds to a certain uniquely defined $\left(\mathbb{C}, C^{\infty}(N, \mathbb{C})\right)$-Lie subalgebra $P$ of $\Omega^{1}(N, \mathbb{C})_{\{\cdot \cdot \cdot\}}$.

Given a stratified symplectic space $N$, we refer to a $\left(\mathbb{C}, C^{\infty}(X, \mathbb{C})\right)$-Lie subalgebra $P$ of $\Omega^{1}(X, \mathbb{C})_{\{\cdot, \cdot\}}$ as a stratified complex polarization for $N$ if, for every stratum $Y$, under the identification of $\mathrm{T}^{\mathbb{C}} Y$ with its (complex) dual coming from the symplectic structure on that stratum, the $\left(\mathbb{C}, C^{\infty}(Y, \mathbb{C})\right)$-Lie subalgebra $P_{Y}$ of $\Omega^{1}(Y, \mathbb{C})_{\{\cdot, \cdot\}}$ generated by the restriction of $P$ to $Y$ is identified with the space of sections of an ordinary complex polarization. A stratified complex polarization is, then, a Kähler polarization provided on any stratum it comes from an ordinary (not necessarily positive) Kähler polarization. We say that a stratified Kähler polarization is complex analytic provided it is induced from a complex analytic structure on $N$, and we define a complex analytic stratified Kähler structure to be a normal Kähler structure provided the complex analytic structure is normal. A normal Kähler structure is positive provided it is positive on each stratum. See Section 2 of [39] for more details.

Let $G$ be a compact Lie group, denote its complex form by $G^{\mathbb{C}}$, and recall the following, cf. Proposition 4.2 of [39].

Proposition 5.1. Given a positive Kähler manifold $N$ with a holomorphic $G^{\mathbb{C}}$ action whose restriction to $G$ preserves the Kähler structure and is hamiltonian, the Kähler polarization $F$ induces a positive normal (complex analytic stratified) Kähler polarization $P^{\mathrm{red}}$ on the reduced space $N^{\mathrm{red}}$, the latter being endowed with its stratified symplectic Poisson algebra $\left(C^{\infty}\left(N^{\mathrm{red}}\right),\{\cdot, \cdot\}^{\mathrm{red}}\right)$.

Under these circumstances, the underlying complex analytic structure of $N^{\text {red }}$ is that of a geometric invariant theory quotient; the existence thereof may be found in [47] and [49]. The existence problem of this complex analytic structure may be seen as one of descent.

\section{Examples}

We will now illustrate the notions introduced so far by means of a number of examples. The interested reader will find more details in [39].

EXAmPle 6.1. For $\ell \geq 1$, consider the constrained system of $\ell$ particles in $\mathbb{R}^{s}$ with total angular momentum zero. Its unreduced phase space $N$ is a product $\left(\mathrm{T}^{*} \mathbb{R}^{s}\right)^{\ell}$ of $\ell$ copies of $\mathrm{T}^{*} \mathbb{R}^{s}$, and we write the points of $N$ in the form $\left(\mathbf{q}_{1}, \mathbf{p}_{1}, \ldots, \mathbf{q}_{\ell}, \mathbf{p}_{\ell}\right)$. Let $H=\mathrm{O}(s, \mathbb{R})$. With reference to the obvious $H$-symmetry, the momentum mapping 
of this system has the form

$$
\mu: N \rightarrow \mathfrak{h}^{*}, \quad \mu\left(\mathbf{q}_{1}, \mathbf{p}_{1}, \ldots, \mathbf{q}_{\ell}, \mathbf{p}_{\ell}\right)=\mathbf{q}_{1} \wedge \mathbf{p}_{1}+\cdots+\mathbf{q}_{\ell} \wedge \mathbf{p}_{\ell}
$$

where the Lie algebra $\mathfrak{h}=\mathfrak{s o}(s, \mathbb{R})$ is identified with its dual in the standard fashion. To elucidate the reduced space, observe that the assignment to $(\mathbf{q}, \mathbf{p})=\left(\mathbf{q}_{1}, \mathbf{p}_{1}, \ldots, \mathbf{q}_{\ell}, \mathbf{p}_{\ell}\right)$ of the real symmetric $(2 \ell \times 2 \ell)$-matrix $\xi(\mathbf{q}, \mathbf{p})=\left[\begin{array}{ll}\mathbf{q}_{j} \mathbf{q}_{k} & \mathbf{q}_{j} \mathbf{p}_{k} \\ \mathbf{p}_{j} \mathbf{q}_{k} & \mathbf{p}_{j} \mathbf{p}_{k}\end{array}\right]_{1 \leq j, k \leq \ell}$ yields a real algebraic map $\xi: N \rightarrow \mathrm{S}_{\mathbb{R}}^{2}\left[\mathbb{R}^{2 \ell}\right]$ from $N$ to the real vector space $\mathrm{S}_{\mathbb{R}}^{2}\left[\mathbb{R}^{2 \ell}\right]$ of real symmetric $(2 \ell \times 2 \ell)$-matrices which passes to an embedding of the reduced space $N^{\text {red }}=\mu^{-1}(0) / H$ into $\mathrm{S}_{\mathbb{R}}^{2}\left[\mathbb{R}^{2 \ell}\right]$, in fact, realizes $N^{\text {red }}$ as a semi-algebraic set in $\mathrm{S}_{\mathbb{R}}^{2}\left[\mathbb{R}^{2 \ell}\right]$. Let $J$ be the standard complex structure on $\mathbb{R}^{2 \ell}$. Now, on the one hand, the association $S \mapsto J S$ identifies $\mathrm{S}_{\mathbb{R}}^{2}\left[\mathbb{R}^{2 \ell}\right]$ with $\mathfrak{s p}(\ell, \mathbb{R})$ in an $\operatorname{Sp}(\ell, \mathbb{R})$-equivariant fashion (with reference to the obvious actions) and hence identifies $N^{\text {red }}$ with a subset of $\mathfrak{s p}(\ell, \mathbb{R})$ which, as observed in [54], is the closure of a certain nilpotent orbit which has been identified as a holomorphic nilpotent orbit in [39]. The Killing form transforms the Lie-Poisson structure on $\mathfrak{s p}(\ell, \mathbb{R})^{*}$ to a Poisson structure on $\mathfrak{s p}(\ell, \mathbb{R})$ which, restricted to $N^{\text {red }}$, yields a stratified symplectic structure. Another observation in [54] entails that this stratified symplectic structure coincides with the Sjamaar-Lerman stratified symplectic structure [70] mentioned earlier arising by symplectic reduction from $N=\left(\mathrm{T}^{*} \mathbb{R}^{s}\right)^{\ell}$. We mention in passing that, $\mathfrak{s p}(\ell, \mathbb{R})$ being identified with its dual by means of an appropriate positive multiple of the Killing form, as well as with $S_{\mathbb{R}}^{2}\left[\mathbb{R}^{2 \ell}\right]$, the map $\xi$ is essentially the momentum mapping for the obvious $\operatorname{Sp}(\ell, \mathbb{R})$-action on $N$.

On the other hand, the choice of $J$ determines a maximal compact subalgebra of $\mathfrak{s p}(\ell, \mathbb{R})$ which is just a copy of $\mathfrak{u}(\ell)$ and, furthermore, a Cartan decomposition $\mathfrak{s p}(\ell, \mathbb{R})=\mathfrak{u}(\ell) \oplus \mathfrak{p}$. Now matrix multiplication by $J$ from the left induces a complex structure on $\mathfrak{p}$ and, with this structure, as a complex vector space, $\mathfrak{p}$ amounts to the complex symmetric square $\mathrm{S}_{\mathbb{C}}^{2}\left[\mathbb{C}^{\ell}\right]$ on $\mathbb{C}^{\ell}$. In particular, orthogonal projection to $\mathfrak{p}$ induces a linear surjection of real vector spaces from $S_{\mathbb{R}}^{2}\left[\mathbb{R}^{2 \ell}\right]$ to $S_{\mathbb{C}}^{2}\left[\mathbb{C}^{\ell}\right]$, uniquely determined by $J$; it is given by the assignment to a real symmetric $(2 \ell \times 2 \ell)$-matrix of the corresponding complex symmetric $(\ell \times \ell)$-matrix with respect to the standard complex structure $J$ on $\mathbb{R}^{2 \ell}$. This projection, restricted to $N^{\text {red }}$, is injective and yields a complex analytic structure on $N^{\text {red }}$. The two structures are compatible and yield a normal (complex analytic stratified) Kähler structure on $N^{\text {red }}$; see [39] for details. We will describe the requisite (complex analytic) stratified Kähler polarization $P$ shortly. For $\ell \geq s$, as a complex analytic space, $N^{\text {red }}$ comes down to $\mathrm{S}_{\mathbb{C}}^{2}\left[\mathbb{C}^{\ell}\right]$ whereas, for $\ell<s$, as a complex analytic space, $N^{\text {red }}$ may be described as a complex determinantal variety in $\mathrm{S}_{\mathbb{C}}^{2}\left[\mathbb{C}^{\ell}\right]$, that is, as an affine variety given by determinantal equations; see e. g. [8] for determinantal varieties. This may be deduced from standard geometric invariant theory results combined with the standard description of the invariants of the classical groups which, in turn, may be found e. g. in [78]. As a stratified symplectic space, the singularity structure of $N^{\text {red }}$ is finer than that of the complex analytic structure, though: Once $\ell$ is fixed, for $s=\ell$, the smooth structure $C^{\infty}\left(N_{\ell}\right)$ and hence the Poisson structure on $N_{\ell}=N^{\text {red }} \cong \mathbb{C}^{d}, d=\frac{\ell(\ell+1)}{2}$, is not standard and, as a stratified symplectic space, $N_{\ell}$ has $\ell+1$ strata. For $s<\ell$, the space $N^{\text {red }}=N_{s}$ (say) may be described as the closure of a stratum in $N_{\ell}$; moreover, a system of $\ell$ particles in $\mathbb{R}^{s-1}$ being viewed as a system of $\ell$ particles in 
$\mathbb{R}^{s}$ via the standard inclusion of $\mathbb{R}^{s-1}$ into $\mathbb{R}^{s}$ yields an injection of $N_{s-1}$ into $N_{s}$. Thus we get a sequence

$$
\{o\}=N_{0} \subseteq N_{1} \subseteq \ldots N_{s-1} \subseteq N_{s} \subseteq \cdots \subseteq N_{\ell}
$$

of injections of normal (complex analytic stratified) Kähler spaces in such a way that, for $1 \leq s \leq \ell, N_{s-1} \subseteq N_{s}$ is the singular locus of $N_{s}$ in the sense of stratified symplectic spaces, and the stratified Kähler structure on $N_{s}$, in particular the requisite Poisson structure, is then simply obtained by restriction from $N_{\ell}$. For example, for $\ell=1,\left(N_{1}, C^{\infty}\left(N_{1}\right),\{\cdot, \cdot\}\right)$ is just the reduced space and reduced Poisson algebra of a system of a single particle in $\mathbb{R}^{n}(n \geq 2)$ with angular momentum zero explained in the Example 4.2 above. For $\ell=s=2$, the space $N_{2}=N^{\text {red }}$ is complex analytically a copy of $\mathbb{C}^{3}$ which, as a stratified symplectic space, sits inside $\mathfrak{s p}(2, \mathbb{R})$, and we need ten generators to describe the Poisson structure on $N_{2}$. The reduced space $N_{1}$ for $\ell=2, s=1$ is here complex analytically realized inside $N_{2} \cong \mathbb{C}^{3}$ as the quadric $Y^{2}=X Z$.

To introduce coordinates, and to spell out a description of the complex analytic stratified Kähler polarizations, consider the complexification $\mathfrak{s p}(\ell, \mathbb{C})$ of $\mathfrak{s p}(\ell, \mathbb{R})$; this complexification sits inside the complex polynomial algebra $\mathbb{C}\left[z_{1}, \ldots, z_{\ell}, \bar{z}_{1}, \ldots, \bar{z}_{\ell}\right]$ as its homogeneous quadratic constituent. The complexification $\mathfrak{k}^{\mathbb{C}} \cong \mathfrak{g l}(\ell, \mathbb{C})$ of the maximal compact subalgebra $\mathfrak{k}=\mathfrak{u}(\ell)$ of $\mathfrak{s p}(\ell, \mathbb{R})$ is the span of the $z_{j} \bar{z}_{k}$ 's and, with reference to the Cartan decomposition $\mathfrak{s p}(\ell, \mathbb{R})=\mathfrak{u}(\ell) \oplus \mathfrak{p}$ of $\mathfrak{s p}(\ell, \mathbb{R})$, the constituents $\mathfrak{p}^{+}$and $\mathfrak{p}^{-}$of the decomposition $\mathfrak{p}^{\mathbb{C}}=\mathfrak{p}^{+} \oplus \mathfrak{p}^{-}$are the spans of the $z_{j} z_{k}$ 's and the $\bar{z}_{j} \bar{z}_{k}$ 's, respectively; this gives an explicit description of $\mathfrak{p}^{+}$and $\mathfrak{p}^{-}$as $\mathrm{S}_{\mathbb{C}}^{2}\left[\mathbb{C}^{\ell}\right]$ and $\overline{S_{\mathbb{C}}^{2}\left[\mathbb{C}^{\ell}\right]}$, respectively. Furthermore, $\mathfrak{k}=\mathfrak{u}(\ell)$ sits inside $\mathfrak{s p}(\ell, \mathbb{C})$ as the real span of the $z_{j} \bar{z}_{k}+\bar{z}_{j} z_{k}$ 's and $i\left(z_{j} \bar{z}_{k}-\bar{z}_{j} z_{k}\right)$ 's, and $\mathfrak{p}$ sits inside $\mathfrak{s p}(\ell, \mathbb{C})$ as the real span of the $z_{j} z_{k}+\bar{z}_{j} \bar{z}_{k}$ 's and $i\left(z_{j} z_{k}-\bar{z}_{j} \bar{z}_{k}\right)$ 's; the assignment to a real symmetric $(2 \ell \times 2 \ell)$-matrix of the corresponding complex symmetric $(\ell \times \ell)$-matrix is given by the association

$$
z_{j} z_{k}+\bar{z}_{j} \bar{z}_{k} \longmapsto z_{j} z_{k}, \quad i\left(z_{j} z_{k}-\bar{z}_{j} \bar{z}_{k}\right) \longmapsto i z_{j} z_{k}
$$

The summands $\mathfrak{p}^{+}$and $\mathfrak{p}^{-}$are the irreducible $\mathfrak{k}^{\mathbb{C}}$-representations in $\mathfrak{s p}(\ell, \mathbb{C})$ complementary to $\mathfrak{k}^{\mathbb{C}}$.

The homogeneous quadratic polynomials in the variables $z_{1}, \ldots, z_{\ell}, \bar{z}_{1}, \ldots, \bar{z}_{\ell}$ yield coordinates on $\mathfrak{s p}(\ell, \mathbb{R})$ and hence, via restriction, on $N^{\text {red }}$, that is, the smooth structure $C^{\infty}\left(N^{\text {red }}, \mathbb{C}\right)$ may be described as the algebra of smooth functions in these variables, subject to the relations coming from the embedding of $N^{\text {red }}$ into $\mathfrak{s p}(\ell, \mathbb{R})$. Now, the differentials $d\left(z_{j} z_{k}\right)$ of the coordinate functions $z_{j} z_{k}(1 \leq j, k \leq \ell)$ (that is, of those coordinate functions which do not involve any of the $\bar{z}_{j}$ 's) generate the corresponding complex analytic stratified Kähler polarization $P \subseteq \Omega^{1}\left(N^{\text {red }}, \mathbb{C}\right)$ as an $\left(\mathbb{C}, C^{\infty}\left(N^{\text {red }}, \mathbb{C}\right)\right)$-Lie subalgebra of $\Omega^{1}\left(N^{\text {red }}, \mathbb{C}\right)_{\{\cdot,\}}$.

In [39], we developed a theory of holomorphic nilpotent orbits of hermitian Lie algebras and established the fact that the (topological) closure of any holomorphic nilpotent orbit inherits a normal (complex analytic stratified) Kähler structure. The space $N^{\text {red }}$, realized as the closure of a holomorphic nilpotent orbit in $\mathfrak{s p}(\ell, \mathbb{R})$, is a special case thereof.

EXAMPLE 6.2. A variant of the above example arises from the constrained system of $\ell$ harmonic oscillators in $\mathbb{R}^{s}$ with total angular momentum zero and constant 
energy. Its unreduced phase space $Q$ is a copy of complex projective space $\mathbb{P}^{s \ell-1} \mathbb{C}$ of complex dimension $s \ell-1$. For $\ell \geq s$, as a complex analytic space, $Q^{\text {red }}$ coincides with the (complex) projectivization $\mathbb{P S}_{\mathbb{C}}^{2}\left[\mathbb{C}^{\ell}\right]$ of $\mathrm{S}_{\mathbb{C}}^{2}\left[\mathbb{C}^{\ell}\right]$ whereas for $\ell<s$, as a complex analytic space, $Q^{\text {red }}$ may be described as a complex projective determinantal variety in $\mathbb{P S}_{\mathbb{C}}^{2}\left[\mathbb{C}^{\ell}\right]$. In fact, the determinantal equations mentioned in Example 6.1 above are homogeneous and yield the requisite homogeneous equations for the present case. In the same vein as before, we get a sequence

$$
Q_{1} \subseteq \ldots Q_{s-1} \subseteq Q_{s} \subseteq \cdots \subseteq Q_{\ell} \cong \mathbb{P}^{d} \mathbb{C}, \quad d=\frac{\ell(\ell+1)}{2}-1
$$

of injections of compact normal (complex analytic stratified) Kähler spaces in such a way that, for $2 \leq s \leq \ell, Q_{s-1} \subseteq Q_{s}$ is the singular locus of $Q_{s}$ in the sense of stratified symplectic spaces, each $Q_{s}$ being the closure of a stratum in $Q_{\ell}$, and the stratified Kähler structure on $Q_{s}$, in particular the requisite Poisson structure, is simply obtained by restriction from $Q_{\ell}$. Complex analytically, each $Q_{s}$ is a projective variety. Again, the smooth structure $C^{\infty}\left(Q_{\ell}\right)$ and hence the Poisson structure on $Q_{\ell} \cong \mathbb{P}^{d} \mathbb{C}(s=\ell)$ is not the standard one (which arises from the Fubini-Study metric on complex projective space) and, as a stratified symplectic space, $Q_{\ell}$ has $\ell$ strata. For example, for $\ell=s=2$, the space $Q_{2}$ is complex analytically a copy of $\mathbb{P}^{2} \mathbb{C}$, and the corresponding reduced space $Q_{1}$ (for $\ell=2, s=1$ ), which is abstractly just complex projective 1-space, sits complex analytically inside $Q_{2} \cong \mathbb{P}^{2} \mathbb{C}$ as the projective conic $Y^{2}=X Z$. These spaces are particular cases of a systematic class of examples of exotic projective varieties, introduced and explored in our paper [39].

REMARK 6.3. Given a Lie group $G$, a smooth hamiltonian $G$-space, and a real $G$-invariant polarization, the question arises whether the statement of Proposition 5.1 still holds for this real polarization. When we try to identify, on the reduced level, a stratified version of such a polarization, we may run into the following difficulty, though: Under the circumstances of the Example 6.1 , let $\ell=1$, and consider the vertical polarization on $N=\mathrm{T}^{*} \mathbb{R}^{n}$. This polarization integrates to the foliation-even fibration-defined by the projection map from $\mathrm{T}^{*} \mathbb{R}^{n}$ to $\mathbb{R}^{n}$. This foliation is clearly $\mathrm{O}(n, \mathbb{R})$-invariant and, in terms of the standard coordinates $\mathbf{q}=\left(q^{1}, \ldots, q^{n}\right)$ on $\mathbb{R}^{n}$, a leaf is given by the equation $\mathbf{q}=\mathbf{q}_{0}$ where $\mathbf{q}_{0}$ is a constant. We will now write the ordinary scalar product of two vectors $\mathbf{x}$ and $\mathbf{y}$ as $\mathbf{x y}$. With these preparations out of the way, under the present circumstances, the assignment to $(\mathbf{q}, \mathbf{p}) \in \mathrm{T}^{*} \mathbb{R}^{n}$ of $x_{1}=\mathbf{q q}-\mathbf{p p}$ and $x_{2}=2 \mathbf{q p}$ yields a map from $\mathrm{T}^{*} \mathbb{R}^{n}$ to the plane $\mathbb{R}^{2}$ which induces an isomorphism of stratified symplectic spaces from the reduced space $N^{\text {red }}$ onto the exotic plane described in the Example 4.2. In particular, the radius function $r$ is given by $r=\mathbf{q q}+\mathbf{p p}$. Now $2 \mathbf{q q}=x_{1}+r$ whence, under reduction, the leaf $\mathbf{q}=\mathbf{q}_{0}$ passes to the subspace of the plane given by the equation

$$
x_{1}+r=2 \mathbf{q}_{0} \mathbf{q}_{0}=c(\text { say }) .
$$

For $\mathbf{q}_{0} \neq 0$, in the $\left(x_{1}, x_{2}\right)$-plane, this is just the parabola $x_{2}^{2}+2 c x_{1}=c^{2}$ since $r^{2}=x_{1}^{2}+x_{2}^{2}$ while, for $\mathbf{q}_{0}=0$, it is the non-positive half $x_{1}$-axis. The reason for this degeneracy is that the leaf $\mathbf{q}=0$ is not transverse to the momentum mapping $\mu$ in the sense that, whatever $\mathbf{p} \in \mathbb{R}^{n}, \mu(0, \mathbf{p})=0$ while $\operatorname{ker}(d \mu(0, \mathbf{p}))$ and the tangent space of the leaf at $(0, \mathbf{p})$ do not together span the tangent space of $N$ at $(0, \mathbf{p})$. 
Thus the reduced space is still foliated, but one leaf is singular; however even the restriction of this foliation to the top stratum still has a singular leaf, the negative half $x_{1}$-axis. A little thought reveals that this implies that this foliation cannot result from a stratified real polarization, the notion of stratified real polarization being defined in the same fashion as a stratified complex polarization, except that, on each stratum, the polarization should come down to a real polarization. As a side remark we mention that the assignment to a leaf of its intersection point with the non-negative $x_{1}$-axis identifies the space of leaves with the non-negative $x_{1}$-axis, and the latter in fact coincides with the orbit space $\mathbb{R}^{n} / \mathrm{O}(n, \mathbb{R})$. This description visualizes the exceptional role played by the non-positive $x_{1}$-axis. The distribution parallel to this foliation, though, is given by the hamiltonian vector field of the function $\mathbf{q q}$ in $C^{\infty}\left(N^{\text {red }}\right)$; it has the form

$$
\{\mathbf{q q},-\}=\frac{1}{2}\left\{x_{1}+r,-\right\}=-x_{2} \frac{\partial}{\partial x_{1}}+\left(x_{1}+r\right) \frac{\partial}{\partial x_{2}}
$$

and in particular vanishes on the non-positive half $x_{1}$-axis. The function qq generates a maximal abelian Poisson subalgebra of $\left(C^{\infty}\left(N^{\text {red }}\right),\{\cdot, \cdot\}^{\text {red }}\right)$. This phenomenon is typical for cotangent bundles with a hamiltonian action of a Lie group arising from an action of that group on the base with more than a single orbit type. Thus we see that the question whether a polarization other than a Kähler polarization descends to a stratified polarization on the reduced level leads to certain delicacies, and we do not know to what extent we can interpret it merely as a descent problem.

The question whether, under suitable circumstances so that in particular the reduced space is still a smooth manifold, a real polarization descends has been studied in [21].

\section{Prequantization on spaces with singularities}

To develop prequantization over stratified symplectic spaces and to describe the behaviour of prequantization under reduction, in our paper [41], we introduced stratified prequantum modules over stratified symplectic spaces. A stratified prequantum module is defined in terms of the appropriate Lie-Rinehart algebra and determines what we call a costratified prequantum space but the two notions, though closely related, should not be confused.

Let $N$ be a stratified symplectic space, and let $(A,\{\cdot, \cdot\})$ be its stratified symplectic Poisson algebra; a special case would be the ordinary symplectic Poisson algebra of a smooth symplectic manifold. Consider the extension (2.4) of Lie-Rinehart algebras. Given an $(A \otimes \mathbb{C})$-module $M$, we refer to an $\left(A, \bar{L}_{\{\cdot, \cdot\}}\right)$-module structure $\chi: \bar{L}_{\{\cdot, \cdot\}} \longrightarrow \operatorname{End}_{\mathbb{R}}(M)$ on $M$ as a prequantum module structure for $(A,\{\cdot, \cdot\})$ provided (i) the values of $\chi$ lie in $\operatorname{End}_{\mathbb{C}}(M)$, that is to say, the operators $\chi(a, \alpha)$ are complex linear transformations, and (ii) for every $a \in A, \chi(a, 0)=i a \operatorname{Id}_{M}[30,41]$.

We recall from [29] that the assignment to $a \in A$ of $(a, d a) \in \bar{L}_{\{\cdot, \cdot\}}$ yields a morphism $\iota$ of real Lie algebras from $A$ to $\bar{L}_{\{\cdot, \cdot\}}$; this reduces the construction of Lie algebra representations of the Lie algebra which underlies the Poisson algebra $A$ to the construction of representations of $\bar{L}_{\{\cdot, \cdot\}}$. Thus, for any prequantum module $(M, \chi)$, the composite of $\iota$ with $-i \chi$ is a representation $a \mapsto \widehat{a}$ of the $A$ underlying real Lie algebra on $M$, viewed as a complex vector space, by $\mathbb{C}$-linear operators so 
that the constants in $A$ act by multiplication and so that the Dirac condition holds, even though $M$ does not necessarily carry a Hilbert space structure. These operators are given by the formula

$$
\widehat{a}(x)=\frac{1}{i} \chi(0, d a)(x)+a x, \quad a \in A, \quad x \in M .
$$

For illustration, consider an ordinary quantizable symplectic manifold $(N, \sigma)$, with ordinary prequantum bundle $\zeta: \Lambda \rightarrow N$, that is, $\zeta$ is a complex line bundle with a connection $\nabla$ whose curvature equals $-i \sigma$; the assignments $\chi_{\nabla}(a, 0)=i a \operatorname{Id}_{M}(a \in A)$ and $\chi_{\nabla}(0, \alpha)=\nabla_{\pi \sharp(\alpha)}\left(\alpha \in \Omega^{1}(N)_{\{\cdot, \cdot\}}\right)$ then yield a prequantum module structure

$$
\chi_{\nabla}: \bar{L}_{\{, \cdot,\}} \rightarrow \operatorname{End}_{\mathbb{C}}(M) \subseteq \operatorname{End}_{\mathbb{R}}(M)
$$

for $(A,\{\cdot, \cdot\})$. (Here $\pi^{\sharp}: \Omega^{1}(N) \rightarrow \operatorname{Vect}(N)$ refers to the adjoint of the 2 -form $\pi$ induced by the symplectic Poisson structure, cf. Section 2.) This is just the ordinary prequantization construction in another guise.

As before, consider a general stratified symplectic space $N$, with stratified symplectic Poisson algebra $\left(C^{\infty}(N),\{\cdot, \cdot\}\right)$. For each stratum $Y$, let $\left(C^{\infty}(Y),\{\cdot, \cdot\}^{Y}\right)$ be its symplectic Poisson structure, and let

$$
0 \rightarrow C^{\infty}(Y) \rightarrow \bar{L}_{\{\cdot, \cdot\}^{Y}} \rightarrow \Omega^{1}(Y)_{\{\cdot, \cdot\}^{Y}} \rightarrow 0
$$

be the corresponding extension (2.4) of Lie-Rinehart algebras. As in (1.5) of [39], we define a stratified prequantum module for $N$ to consist of

- a prequantum module $(M, \chi)$ for $\left(C^{\infty}(N),\{\cdot, \cdot\}\right)$, together with,

- for each stratum $Y$, a prequantum module structure $\chi_{Y}$ for $\left(C^{\infty}(Y),\{\cdot, \cdot\}^{Y}\right)$ on $M_{Y}=C^{\infty}(Y) \otimes_{C^{\infty}(N)} M$ in such a way that the canonical linear map of complex vector spaces from $M$ to $M_{Y}$ is a morphism of prequantum modules from $(M, \chi)$ to $\left(M_{Y}, \chi_{Y}\right)$.

Given a stratified prequantum module $(M, \chi)$ for $N$, when $Y$ runs through the strata of $N$, we refer to the system

$$
\left(M_{\bar{Y}}, \chi_{\bar{Y}}: \bar{L}_{\{\cdot, \cdot\} \bar{Y}} \rightarrow \operatorname{End}_{\mathbb{R}}\left(M_{\bar{Y}}\right)\right)
$$

of prequantum modules, together with, for every pair of strata $Y, Y^{\prime}$ such that $Y^{\prime} \subseteq \bar{Y}$, the induced morphism

$$
\left(M_{\bar{Y}}, \chi_{\bar{Y}}\right) \rightarrow\left(M_{\bar{Y}^{\prime}}, \chi_{\bar{Y}^{\prime}}\right)
$$

of prequantum modules, as a costratified prequantum space. More formally: Consider the category $\mathcal{C}_{N}$ whose objects are the strata of $N$ and whose morphisms are the inclusions $Y^{\prime} \subseteq \bar{Y}$. We define a costratified complex vector space on $N$ to be a contravariant functor from $\mathcal{C}_{N}$ to the category of complex vector spaces, and a costratified prequantum space on $N$ to be a costratified complex vector space together with a compatible system of prequantum module structures. Thus a stratified prequantum module $(M, \chi)$ for $\left(N, C^{\infty}(N),\{\cdot, \cdot\}\right)$ determines a costratified prequantum space on $N$; see the (1.4) and (1.5) of [41] for details. 
Theorem 7.2. Given a symplectic manifold $N$ with a hamiltonian action of a compact Lie group $G$, a G-equivariant prequantum bundle $\zeta$ descends to a stratified prequantum module ( $\left.\chi^{\mathrm{red}}, M^{\mathrm{red}}\right)$ for the stratified symplectic space $\left(N^{\mathrm{red}}, C^{\infty}\left(N^{\mathrm{red}}\right),\{\cdot, \cdot\}^{\mathrm{red}}\right)$.

Proof. See Theorem 2.1 of [41].

Thus, phrased in the language of prequantum modules, the relationship between the unreduced and reduced prequantum object may be interpreted as one of descent.

In particular, consider a complex analytic stratified Kähler space $\left(N, C^{\infty}(N),\{\cdot, \cdot\}, P\right)$ (cf. Section 5 above or Section 2 of [39]), and let $(M, \chi)$ be a stratified prequantum module for $\left(C^{\infty}(N),\{\cdot, \cdot\}\right)$. We refer to $(M, \chi)$ as a complex analytic stratified prequantum module provided $M$ is the space of $\left(C^{\infty}(N)\right.$-) sections of a complex $V$-line bundle $\zeta$ on $N$ in such a way that $P$ endows $\zeta$ via $\chi$ with a complex analytic structure. If this happens to be the case, $M^{P}$ necessarily amounts to the space of global sections of the sheaf of germs of holomorphic sections of $\zeta$. See Section 3 of [39].

\section{Kähler quantization and reduction}

Let $G$ be a compact Lie group, let $(N, \sigma, \mu)$ be a hamiltonian $G$-space of the kind as that in the circumstances of Proposition 5.1, and suppose that $N$ is quantizable. Thus $N$ is, in particular, a positive Kähler manifold with a holomorphic $G^{\mathbb{C}}$-action whose restriction to $G$ preserves the Kähler structure and is hamiltonian. Write $P$ for the corresponding Kähler polarization, necessarily $G$-invariant, viewed as a $\left(\mathbb{C}, C^{\infty}(N, \mathbb{C})\right)$-Lie subalgebra of the $\left(\mathbb{C}, C^{\infty}(N, \mathbb{C})\right)$-Lie algebra $\Omega^{1}(N, \mathbb{C})_{\{\cdot, \cdot\}}$, and let $\zeta$ be a prequantum bundle. Via its connection, it acquires a holomorphic structure, and the connection is the unique hermitian connection for a corresponding hermitian structure. The momentum mapping induces, in particular, an infinitesimal action of the Lie algebra $\mathfrak{g}$ of $G$ on $\zeta$ preserving the connection and hermitian structure. Suppose that this action lifts to a $G$-action on $\zeta$ preserving the connection and lifting the $G$-action on $N$. For connected $G$, the assumption that the $G$-action lift to one on $\zeta$ is (well known to be) redundant (since the infinitesimal action is essentially given by the momentum mapping) and it will suffice to replace $G$ by an appropriate covering group if need be. Prequantization turns the space of smooth sections of $\zeta$ into a prequantum module for the ordinary smooth symplectic Poisson algebra of $N$. We write this prequantum module as $M$; it inherits a $G$-action preserving the polarization $P$. Hence the quantum module $M^{P}$, that is, the space $\Gamma(\zeta)$ of global holomorphic sections of $\zeta$, is a complex representation space for $G$. This quantum module is the corresponding unreduced quantum state space, except that there is no Hilbert space structure present yet, and reduction after quantization, for the quantum state spaces, amounts to taking the space $\left(M^{P}\right)^{G}$ of $G$-invariant holomorphic sections.

The projection map from the space of smooth $G$-invariant sections of $\zeta$ to $M^{\text {red }}$ restricts to a linear map

$$
\rho: \Gamma(\zeta)^{G} \rightarrow\left(M^{\mathrm{red}}\right)^{P^{\mathrm{red}}}
$$

of complex vector spaces, defined on the space $\left(M^{P}\right)^{G}=\Gamma(\zeta)^{G}$ of $G$-invariant holomorphic sections of $\zeta$. Here and below $P^{\text {red }}$ refers to the stratified Kähler polarization the existence of which is asserted in Proposition 5.1 above, $M^{\text {red }}$ to 
the prequantum module for the stratified Kähler space mentioned in Theorem 7.2 (without having been made explicit there), and $\left(M^{\text {red }}\right)^{P^{\text {red }}}$ to the $P^{\text {red-invariants; }}$ notice that $P^{\text {red }}$ is, in particular, a Lie algebra whence it makes sense to talk about

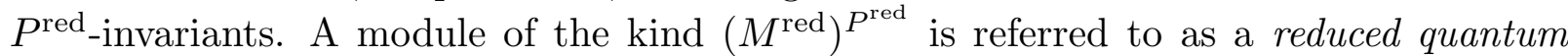
module in [41]. It acquires a costratified Hilbert space structure, the requisite scalar products being induced from appropriate hermitian structures via integration.

As far as the comparison of $G$-invariant unreduced quantum observables and reduced quantum observables is concerned, the statement that Kähler quantization commutes with reduction amounts to the following, cf. Theorem 3.6 in [41].

Theorem 8.2. The data $(N, \sigma, \mu, M, P)$ being fixed so that, in particular, $(N, \sigma, \mu)$ is a smooth hamiltonian $G$-space structure on a quantizable positive Kähler manifold $N$ with a holomorphic $G^{\mathbb{C}}$-action, the Kähler polarization being written as $P$, let $f$ be a smooth $G$-invariant function on $N$ which is quantizable in the sense that it preserves $P$. Then its class $[f] \in C^{\infty}\left(N^{\mathrm{red}}\right)\left(=\left(C^{\infty}(N)\right)^{G} / I^{G}\right)$ is quantizable, i. e. preserves $P^{\text {red }}$ and, for every $h \in\left(M^{P}\right)^{G}$,

$$
\rho(\widehat{f}(h))=\widehat{[f]}(\rho(h))
$$

So far, we did not make any claim to the effect that the reduced quantum module $\left(M^{\text {red }}\right)^{P^{\text {red }}}$ amounts to a space of global holomorphic sections. We now recall that, under the circumstances of Theorem 8.2, the momentum mapping is said to be admissible provided, for every $m \in N$, the path of steepest descent through $m$ is contained in a compact set [68], [49] (§9). For example, when the momentum mapping is proper it is admissible. Likewise, the momentum mapping for a unitary representation of a compact Lie group is admissible in this sense, see Example 2.1 in [68].

The statement "Kähler quantization commutes with reduction" is then completed by the following two observations, cf. [41] ((3.7) and (3.8)).

Proposition 8.3. Under the circumstances of Theorem 8.2, when $\mu$ is admissible and when $N^{\text {red }}$ has a top stratum (i. e. an open dense stratum), for example when $\mu$ is proper, the reduced stratified prequantum module $\left(M^{\mathrm{red}}, \chi^{\mathrm{red}}\right)$ is complex analytic, that is, as a complex vector space, $M^{\mathrm{red}}$ amounts to the space of global holomorphic sections of a suitable holomorphic $V$-line bundle on $N^{\mathrm{red}}$.

The relevant $V$-line bundle on $N^{\text {red }}$ may be found in [68] (Proposition 2.11).

Theorem 8.4. [68] (Theorem 2.15) Under the circumstances of Theorem 8.2, when the momentum mapping $\mu$ is proper, in particular, when $N$ is compact, the map $\rho$ is an isomorphism of complex vector spaces.

In this result, the properness condition, while sufficient, is not necessary, that is, the map $\rho$ may be an isomorphism without the momentum mapping being proper.

A version of Theorem 8.4 has been established in (4.15) of [60]; cf. also [69] and the literature there, as well as [65] and [77] for generalizations to higher dimensional sheaf cohomology.

REMARK 8.5. The statements of Theorems 8.2 and 8.4 are logically independent; in particular the statement of Theorem 8.2 makes sense whether or not $\rho$ is an isomorphism, and its proof does not rely on $\rho$ being an isomorphism. 
Thus we have consistent Kähler quantizations on the unreduced and reduced spaces, including a satisfactory treatment of observables, as indicated by the formula (8.2.1). We have already pointed out in the introduction that examples in finite dimensions abound. We hope that this kind of approach, suitably adapted, will eventually yield the quantization of certain infinite dimensional systems arising from field theory.

REMARK 8.6. KEMPF's descent lemma [46] mentioned earlier characterizes, among the holomorphic $V$-line bundles which arise on a geometric invariant theory quotient by the standard geometric invariant theory construction, those which are ordinary (holomorphic) line bundles. In the circumstances of Theorem 8.4, complex analytically, the space $N^{\text {red }}$ is a geometric invariant theory quotient, and the $V$-line bundle which underlies the reduced quantum module arises by the standard geometric invariant theory construction. Here the term "descent" is used in its strict sense.

Illustration 8.7. Under the circumstances of the Example 6.2, let $\mathcal{O}(1)$ be the ordinary hyperplane bundle on $Q=\mathbb{P}^{s \ell-1} \mathbb{C}$ and, as usual, for $k \geq 1$, write its $k^{\prime}$ th power as $\mathcal{O}(k)$. The unitary group $\mathrm{U}(s \ell)$ acts on $\mathbb{P}^{s \ell-1} \mathbb{C}$ in a hamiltonian fashion having as momentum mapping the familiar embedding of $\mathbb{P}^{s \ell-1} \mathbb{C}$ into $\mathfrak{u}(s \ell)^{*}$, and the adjoint thereof yields a morphism of Lie algebras from $\mathfrak{u}(s \ell)$ to $C^{\infty}\left(\mathbb{P}^{s \ell-1} \mathbb{C}\right)$, the latter being endowed with its symplectic Poisson structure coming from the Fubini-Study metric. It is a standard fact that, for $k \geq 1$, Kähler quantization, with reference to $k \omega$ and $\mathcal{O}(k)$ (where $\omega$ is the Fubini-Study form), yields the $k$ th symmetric power of the standard representation defining the Lie algebra $\mathfrak{u}(s \ell)$, and this representation integrates to the $k$ 'th symmetric power $E_{s}^{k}$ of the standard representation $E_{s}$ defining the group $\mathrm{U}(s \ell)$. (We use the subscript $-_{s}$ since here and below $\ell$ is fixed while $s$ varies.) The symmetry group $H=\mathrm{O}(s, \mathbb{R})$ of the constrained system in (6.1) above appears as a subgroup of $\mathrm{U}(s \ell)$ in an obvious fashion and, viewed as this subgroup, $H$ centralizes the subgroup $\mathrm{U}(\ell)=\mathrm{Sp}(\ell, \mathbb{R}) \cap \mathrm{U}(s \ell)$ (the maximal compact subgroup $\mathrm{U}(\ell)$ of $\operatorname{Sp}(\ell, \mathbb{R}))$; hence, for $k \geq 1$, the subspace $\left(E_{s}^{k}\right)^{H}$ of $H$-invariants is a $\mathrm{U}(\ell)$-representation. On the other hand, with an abuse of notation, let $\mathcal{O}(1)$ be the hyperplane bundle on the reduced space $Q_{\ell}=\mathbb{P}^{d} \mathbb{C}, d=\frac{\ell(\ell+1)}{2}-1$ and, for $k \geq 1$, let $\mathcal{O}(k)$ be its $k^{\prime}$ th power. The space of holomorphic sections thereof, $\Gamma(\mathcal{O}(k))$, amounts to the $k^{\prime}$ th symmetric power $S_{\mathbb{C}}^{k}\left[\mathfrak{p}^{*}\right]$ of the dual of $\mathfrak{p}=\mathrm{S}_{\mathbb{C}}^{2}\left[\mathbb{C}^{\ell}\right]$ (the space of homogeneous degree $k$ polynomial functions on $\mathfrak{p})$. For $1 \leq s \leq \ell$ and $k \geq 1$, maintain the notation $\mathcal{O}(k)$ for the restriction of the $k^{\prime}$ th power of the hyperplane bundle to $Q_{s} \subseteq Q_{\ell}$; for $s<\ell$, the space of holomorphic sections $\widetilde{E}_{s}^{k}$ of $\mathcal{O}(k)$ is now a certain quotient of $\widetilde{E}_{\ell}^{k}=\mathrm{S}_{\mathbb{C}}^{k}\left[\mathfrak{p}^{*}\right]$ which will be made precise below.

For $1 \leq s \leq \ell$, the composite of the embedding of $N_{s}$ into $\mathfrak{s p}(\ell, \mathbb{R})^{*}$ with the surjection from $\mathfrak{s p}(\ell, \mathbb{R})^{*}$ to $\mathfrak{u}(\ell)^{*}$ induced from the injection of $\mathfrak{u}(\ell)$ into $\mathfrak{s p}(\ell, \mathbb{R})$ yields a map from $N_{s}$ to $\mathfrak{u}(\ell)^{*}$ which descends to a map from $Q_{s}$ to $\mathfrak{u}(\ell)^{*}$, the adjoint of which induces a morphism of Lie algebras from $\mathfrak{u}(\ell)$ to $C^{\infty}\left(Q_{s}\right)$, the latter being endowed with its stratified symplectic Poisson structure explained earlier. For $k \geq 1$, the space of sections $M^{\text {red }}$ (cf. Theorem 7.2) of $\mathcal{O}(k)$, with reference to a $C^{\infty}\left(Q_{s}\right)$-module structure constructed in [41] and not made precise here, inherits a stratified prequantum module structure; and stratified Kähler quantization yields a $\mathrm{U}(\ell)$-representation on the space $\widetilde{E}_{s}^{k}$, which amounts to that written earlier as $\left(M^{\mathrm{red}}\right)^{P^{\text {red }}}$, cf. (8.1), in such a way that the map $\rho$ given as (8.1) above identifies the representation written above as $\left(E_{s}^{2 k}\right)^{H}$ with $\widetilde{E}_{s}^{k}$; moreover, the spaces $\left(E_{s}^{2 k-1}\right)^{H}$ 
are zero.

We conclude with an explicit description of the spaces $\left(E_{s}^{2 k}\right)^{H}$ or, equivalently, of the spaces $\widetilde{E}_{s}^{k}$ : Introduce coordinates $x_{1}, \ldots, x_{\ell}$ on $\mathbb{C}^{\ell}$. These give rise to coordinates $\left\{x_{i, j}=x_{j, i} ; 1 \leq i, j \leq \ell\right\}$ on $\mathfrak{p}=S_{\mathbb{C}}^{2}\left[\mathbb{C}^{\ell}\right]$, and the determinants

$$
\delta_{1}=x_{1,1}, \quad \delta_{2}=\left|\begin{array}{ll}
x_{1,1} & x_{1,2} \\
x_{1,2} & x_{2,2}
\end{array}\right|, \quad \delta_{3}=\left|\begin{array}{lll}
x_{1,1} & x_{1,2} & x_{1,3} \\
x_{1,2} & x_{2,2} & x_{2,3} \\
x_{1,3} & x_{2,3} & x_{3,3}
\end{array}\right|, \quad \text { etc. }
$$

are highest weight vectors for certain $\mathrm{U}(\ell)$-representations. For $1 \leq s \leq r$ and $k \geq 1$, the $\mathrm{U}(\ell)$-representation $\widetilde{E}_{s}^{k}$ is the sum of the irreducible representations having as highest weight vectors the monomials

$$
\delta_{1}^{\alpha} \delta_{2}^{\beta} \ldots \delta_{s}^{\gamma}, \quad \alpha+2 \beta+\cdots+s \gamma=k,
$$

and the morphism from $\widetilde{E}_{s}^{k}$ to $\widetilde{E}_{s-1}^{k}$ coming from restriction from $Q_{s}$ to $Q_{s-1}$ is an isomorphism on the span of those irreducible representations which do not involve $\delta_{s}$ and has the span of the remaining ones as its kernel. In particular, this explains

how $\widetilde{E}_{s}^{k}$ arises from $\widetilde{E}_{\ell}^{k}=\mathrm{S}_{\mathbb{C}}^{k}\left[\mathfrak{p}^{*}\right]$. For $1 \leq s \leq \ell$, the $\operatorname{system}\left(\widetilde{E}_{1}^{k}, \widetilde{E}_{2}^{k}, \ldots, \widetilde{E}_{s}^{k}\right)$ is an example of a costratified quantum space.

The alerted reader is invited to consult [41] for more details.

\section{REFERENCES}

1. R. Abraham and J. E. Marsden, Foundations of Mechanics, Benjamin/Cummings Publishing Company, 1978.

2. R. Almeida and A. Kumpera, Structure produit dans la catégorie des algébrö̈des de Lie, An. Acad. Brasil. Cienc. 53 (1981), 247-250.

3. J. M. Arms, R. Cushman, and M. J. Gotay, A universal reduction procedure for Hamiltonian group actions, in: The geometry of Hamiltonian systems, T. Ratiu, ed., MSRI Publ. 20 (1991), Springer-Verlag, Berlin · Heidelberg · New York · Tokyo, 33-51.

4. J. M. Arms, J. E. Marsden, and V. Moncrief, Symmetry and bifurcation of moment mappings, Comm. Math. Phys. 78 (1981), 455-478.

5. M. F. Atiyah, Complex analytic connections in fibre bundles, Trans. Amer. Math. Soc. 85 (1957), 181-207.

6. A. A. Beilinson and V. V. Schechtmann, Determinant bundles and Virasoro algebras, Comm. Math. Physics 118 (1988), 651-701.

7. R. Bkouche, Structures (K, A)-linéaires, C. R. A. S. Paris Série A 262 (1966), $373-376$.

8. W. Bruns and U. Vetter, Determinantal Rings, Lecture Notes in Mathematics, Vol. 1327, Springer-Verlag, Berlin · Heidelberg · New York, 1988.

9. Ana Canas da Silva and Alan Weinstein, Geometric models for Noncommutative Algebras, Berkeley Mathematical Lecture Notes, Vol. 10, AMS, Boston Ma, 1999.

10. S. U. Chase, Group scheme actions by inner automorphisms, Comm. Alg. 4 (1976), 403-434. 
11. S. Chemla, A duality property for complex Lie algebroids, Math. Z. 232 (1999), $367-388$.

12. C. Chevalley and S. Eilenberg, Cohomology theory of Lie groups and Lie algebras, Trans. Amer. Math. Soc. 63 (1948), 85-124.

13. M. Crainic and R. L. Fernandes, Integrability of Lie brackets, Ann. of Math. (to appear), math.DG/0105033.

14. C. M. de Barros, Espaces infinitésimaux, Cahiers Topologie Géom. différentielle 7 (1964AA).

15. P. A. M. Dirac, Lectures on Quantum Mechanics, Belfer Graduate School of Science, Yeshiva University, New York, 1964.

16. P. A. M. Dirac, Generalized Hamiltonian systems, Can. J. of Math. 12 (1950), $129-148$.

17. J.-M. Drezet and M.S. Narasimhan, Groupe de Picard des variétés de modules de fibrés semistables sur les courbes algébriques, Invent. Math. 97 (1989), 53-94.

18. A. Fahim, Extensions galoisiennes d'algèbres différentielles, Pacific J. Math. 180 (1997), 7-40.

19. B. Fuchssteiner, The Lie algebra structure of degenerate Hamiltonian and bihamiltonian systems, Progr. Theor. Phys. 68 (1982), 1082-1104.

20. M. Goresky and R. MacPherson, Intersection homology theory, Topology 19 (1980), 135-162.

21. M. J. Gotay, Constraints, reduction, and quantization, J. of Math. Phys. 27 (1986), 2051-2066.

22. V. W. Guillemin and S. Sternberg, Geometric quantization and multiplicities of group representations, Invent. Math. 67 (1982), 515-538.

23. R. Hermann, Analytic continuations of group representations. IV., Comm. Math. Phys. 5 (1967), 131-156.

24. J. Herz, Pseudo-algèbres de Lie, C. R. Acad. Sci. Paris 236 (1953), 1935-1937.

25. P. J. Higgins and K. Mackenzie, Algebraic constructions in the category of Lie algebroids, J. of Algebra 129 (1990), 194-230.

26. V. Hinich and V. Schechtman, Deformation theory and Lie algebra homology. I., alg-geom/9405013, Alg. Colloquium 4:2 (1997), 213-240; II., 291-316.

27. G. Hochschild, Simple algebras with purely inseparable splitting field of exponent 1, Trans. Amer. Math. Soc. 79 (1955), 477-489.

28. G. Hochschild, B. Kostant, and A. Rosenberg, Differential forms on regular affine algebras, Trans. Amer. Math. Soc. 102 (1962), 383-408.

29. J. Huebschmann, Poisson cohomology and quantization, J. für die reine und angewandte Mathematik 408 (1990), 57-113.

30. J. Huebschmann, On the quantization of Poisson algebras, Symplectic Geometry and Mathematical Physics, Actes du colloque en l'honneur de Jean-Marie Souriau, P. Donato, C. Duval, J. Elhadad, G.M. Tuynman, eds.; Progress in Mathematics, Vol. 99 (1991), Birkhäuser-Verlag, Boston · Basel • Berlin, $204-233$.

31. J. Huebschmann, Poisson geometry of certain moduli spaces, Lectures delivered at the "14th Winter School", Srni, Czeque Republic, January 1994, Rendiconti del Circolo Matematico di Palermo, Serie II 39 (1996), 15-35.

32. J. Huebschmann, On the Poisson geometry of certain moduli spaces, in: Proceedings of an international workshop on "Lie theory and its applications in 
physics", Clausthal, 1995, H. D. Doebner, V. K. Dobrev, J. Hilgert, eds. (1996), World Scientific, Singapore · New Jersey · London · Hong Kong, 89-101.

33. J. Huebschmann, Lie-Rinehart algebras, Gerstenhaber algebras, and BatalinVilkovisky algebras, Annales de l'Institut Fourier 48 (1998), 425-440, math.DG/9704005.

34. J. Huebschmann, Extensions of Lie-Rinehart algebras and the Chern-Weil construction, Festschrift in honour of Jim Stasheff's 60'th anniversary, Cont. Math. 227 (1999), 145-176, math.DG/9706002.

35. J. Huebschmann, Duality for Lie-Rinehart algebras and the modular class, Journal für die reine und angew. Math. 510 (1999), 103-159, math.DG/9702008.

36. J. Huebschmann, Differential Batalin-Vilkovisky algebras arising from twilled Lie-Rinehart algebras, Banach center publications 51 (2000), 87-102.

37. J. Huebschmann, Singularities and Poisson geometry of certain representation spaces, in: Quantization of Singular Symplectic Quotients, N. P. Landsman, M. Pflaum, M. Schlichenmaier, eds., Workshop, Oberwolfach, August 1999, Progress in Mathematics, Vol. 198 (2001), Birkhäuser-Verlag, Boston · Basel • Berlin, 119-135, math.DG/0012184.

38. J. Huebschmann, Twilled Lie-Rinehart algebras and differential Batalin-Vilkovisky algebras, math.DG/9811069.

39. J. Huebschmann, Kähler spaces, nilpotent orbits, and singular reduction, math.DG/0104213.

40. J. Huebschmann, Severi varieties and holomorphic nilpotent orbits, math.DG/0206143.

41. J. Huebschmann, Kähler reduction and quantization, math.SG/0207166.

42. L. Illusie, Complexe cotangent et déformations. II, Lecture Notes in Mathematics No. 238, Springer-Verlag, Berlin · Heidelberg · New York, 1972.

43. N. Jacobson, An extension of Galois theory to non-normal and non-separable fields, Amer. J. Math. 66 (1944), 1-29.

44. F. W. Kamber and $\mathrm{Ph}$. Tondeur, Invariant differential operators and the cohomology of Lie algebra sheaves, Memoirs of the Amer. Math. Soc. 113 (1971), Amer. Math. Soc., Providence, R. I.

45. D. Kastler and R. Stora, A differential geometric setting for BRS transformations and anomalies. I.II., J. Geom. Phys. 3 (1986), 437-482; 483-506.

46. G. Kempf, Instability in invariant theory, Ann. of Math. 108 (1978), 299-316.

47. G. Kempf and L. Ness, The length of vectors in representation spaces, Algebraic geometry, Copenhagen, 1978, Lecture Notes in Mathematics 732 (1978), Springer-Verlag, Berlin · Heidelberg · New York, 233-244.

48. A. A. Kirillov, Unitary representations of nilpotent Lie groups, Uspehi Mat. Nauk. 17 (1962), 57-101; Russ. Math. Surveys 17 (1962), 57-101.

49. F. Kirwan, Cohomology of quotients in symplectic and algebraic geometry, Princeton University Press, Princeton, New Jersey, 1984.

50. Y. Kosmann-Schwarzbach and F. Magri, Poisson-Nijenhuis structures, Annales Inst. H. Poincaré Série A (Physique théorique) 53 (1989), 35-81.

51. B. Kostant, Quantization and unitary representations, In: Lectures in Modern Analysis and Applications, III, ed. C. T. Taam, Lecture Notes in Math. 170 (1970), Springer-Verlag, Berlin · Heidelberg • New York, 87-207. 
52. B. Kostant and S. Sternberg, Anti-Poisson algebras and current algebras, unpublished manuscript (1990).

53. I. S. Krasil'shchik, V. V. Lychagin, and A. M. Vinogradov, Geometry of Jet Spaces and Nonlinear Partial Differential Equations, Advanced Studies in Contemporary Mathematics, vol. 1, Gordon and Breach Science Publishers, New York, London, Paris, Montreux, Tokyo, 1986.

54. E. Lerman, R. Montgomery and R. Sjamaar, Examples of singular reduction, Symplectic Geometry, Warwick, 1990, D. A. Salamon, editor, London Math. Soc. Lecture Note Series 192 (1993), Cambridge University Press, Cambridge, UK, $127-155$.

55. A. Lichnerowicz, Les variétés de Poisson et leurs algèbres de Lie associées, J. Diff. Geo. 12 (1977), 253-300.

56. K. Mackenzie, Lie groupoids and Lie algebroids in differential geometry, London Math. Soc. Lecture Note Series, vol. 124, Cambridge University Press, Cambridge, England, 1987.

57. K. Mackenzie, Lie algebroids and Lie pseudoalgebras, Bull. London Math. Soc. 27 (2) (1995), 97 - 147.

58. J. Marsden and A. Weinstein, Reduction of symplectic manifolds with symmetries, Rep. on Math. Phys. 5 (1974), 121-130.

59. W. S. Massey and F. P. Petersen, The cohomology structure of certain fibre spaces.I, Topology 4 (1965), 47-65.

60. M. S. Narasimhan and T. R. Ramadas, Factorization of generalized theta functions, Inventiones 114 (1993), 565-623.

61. E. Nelson, Tensor Analysis, Princeton University Press, Princeton, N. J., 1967.

62. R. S. Palais, The cohomology of Lie rings, Amer. Math. Soc., Providence, R. I., Proc. Symp. Pure Math. III (1961), 130-137.

63. J. Pradines, Théorie de Lie pour les groupoïdes différentiables. Relations entre propriétés locales et globales, C. R. Acad. Sci. Paris Série A 263 (1966), 907-910.

64. J. Pradines, Théorie de Lie pour les groupoïdes différentiables. Calcul différentiel dans la catégorie des groupoïdes infinitésimaux, C. R. Acad. Sci. Paris Série A 264 (1967), 245-248.

65. T. R. Ramadas, Factorization of generalised theta functions II: The Verlinde formula, Topology 35 (1996), 641-654.

66. G. Rinehart, Differential forms for general commutative algebras, Trans. Amer. Math. Soc. 108 (1963), 195-222.

67. I. E. Segal, Quantization of non-linear systems, J. of Math. Phys. 1 (1960), $468-488$.

68. R. Sjamaar, Holomorphic slices, symplectic reduction, and multiplicities of representations, Ann. of Math. 141 (1995), 87-129.

69. R. Sjamaar, Symplectic reduction and Riemann-Roch formulas for multiplicities, Bull. Amer. Math. Soc. 33 (1996), 327-338.

70. R. Sjamaar and E. Lerman, Stratified symplectic spaces and reduction, Ann. of Math. 134 (1991), 375-422.

71. J. Śniatycki, Geometric quantization and quantum mechanics, Applied Mathematical Sciences No. 30, Springer-Verlag, Berlin · Heidelberg · New York, 1980. 
72. J. Śniatycki, Constraints and quantization, in: Nonlinear partial differential operators and quantization procedures, Clausthal 1981, eds. S. I. Anderson and H. D. Doebner, Lecture Notes in Mathematics, No. 1037 (1983), Springer-Verlag, Berlin · Heidelberg • New York, 301-334.

73. J. Śniatycki and A. Weinstein, Reduction and quantization for singular moment mappings, Lett. Math. Phys. 7 (1983), 155-161.

74. J. M. Souriau, Quantification géométrique, Comm. Math. Physics 1 (1966), 374-398.

75. J. D. Stasheff, Homological reduction of constrained Poisson algebras, J. of Diff. Geom. 45 (1997), 221-240.

76. N. Teleman, A characteristic ring of a Lie algebra extension, Accad. Naz. Lincei. Rend. Cl. Sci. Fis. Mat. Natur. (8) 52 (1972), 498-506 and 708-711.

77. C. Teleman, The quantization conjecture revisited, Ann. of Math. 152 (2000), $1-43$.

78. H. Weyl, The classical groups, Princeton University Press, Princeton, New Jersey, 1946.

79. N. M. J. Woodhouse, Geometric quantization, Second edition, Clarendon Press, Oxford, 1991.

UStL, UfR de Mathématiques, CNRS-UMR 8524, F-59 655 Villeneuve D’Ascq Cédex, FRANCE

JoHANNES.HUEBSCHMANN@AGAT.UNIV-LILLE1.FR 